\title{
DIMENSIONAMENTO DE PESSOAL DE ENFERMAGEM NA UNIDADE SEMI-INTENSIVA DE UM HOSPITAL UNIVERSITÁRIO DE CURITIBA
}

\author{
Lillian Daisy G. Wolff', Cíntia da Silva Mazur², Cristiane Wiezbicki, Cristina Bello Barros ${ }^{3}$, Valdete A. S. de Quadros ${ }^{4}$
}

\begin{abstract}
RESUMO: Estudo descritivo-exploratório, cujo objetivo foi dimensionar o número de profissionais de enfermagem no Centro de Terapia Semi-Intensiva (CTSI) de um hospital universitário. Os resultados do Nursing Activities Score (NAS) aplicado a 96 pacientes evidenciaram que a carga de trabalho junto a pacientes críticos neurológicos e semicríticos clínicos é maior, e menor para pacientes críticos cardiológicos. A complexidade e a intensidade do cuidado não sofrem influência da idade, mas das condições clínicas e da dependência do paciente em relação à Enfermagem. $\mathrm{O}$ atendimento das necessidades de cuidado dos pacientes requer $41 \%$ de profissionais de enfermagem a mais para o CTSI. O alto Índice de Segurança Técnica para a Unidade deve-se principalmente pela jornada de trabalho de trinta horas semanais. O NAS é um instrumento gerencial que possibilita adequar o número de profissionais às reais necessidades de cuidado dos pacientes, a fim de que este seja realizado de modo seguro e com a qualidade almejada
\end{abstract}

PALAVRAS-CHAVE: Administração de Recursos Humanos; Enfermagem; Cuidados críticos; Carga de trabalho.

\section{MEASURING NURSING WORKLOAD IN THE SEMI-INTENSIVE UNIT OF A TEACHING HOSPITAL IN THE CITY OF CURITIBA/BRAZIL}

\begin{abstract}
This descriptive-exploratory research aimed to estimate the nursing workload in a Semi-Intensive Care Center (SICC) of a university hospital. The results obtained by the Nursing Activities Score (NAS), applied to 96 inpatients, showed that the workload is higher to critical neurological inpatients and to semi-critical clinical ones, and lower to cardiac critical inpatients. The complexity and the intensity of their care were not influenced by age, but by their clinical conditions and dependence on nursing staff. In order to meet patients' caring needs at the SICC, that would request $41 \%$ more nurses. The high Technical Safety Index is due to the 30-hour working period weekly with two dayoffs. NAS is an important managerial instrument which enables to adequate the number of professionals to inpatients' real caring needs, so that safe and qualified care is delivered.
\end{abstract}

KEYWORDS: Personnel Management; Nursing; Critical care; Workload.

\section{DIMENSIÓN DE PERSONAL DE ENFERMERÍA EN LA UNIDAD DE CUIADADOS SEMIINTENSIVOS DE UN HOSPITAL UNIVERSITARIO DE CURITIBA}

RESUMEN: Es un estudio descriptivo y exploratorio, cuyo objetivo fue hazer la dimensión de los profesionales de enfermería en Centro de Cuidado Semiintensivo de un hospital universitario. Los resultados del Nursing Activities Score (NAS) aplicado a 96 pacientes evidenciaron que la carga de trabajo con pacientes críticos neurológicos y semicríticos clínicos es mayor, y menor para pacientes críticos cardiológicos. La complejidad y la intensidad del cuidado no tienen influencia de la edad, pero de las condiciones clínicas y de la dependencia del paciente en relación a la enfermería. Para el atendimiento de las necesidades de cuidado de los pacientes son necesarios $41 \%$ de profesionales de enfermería a más para el Centro de Cuidado. El gran Índice de Seguridad Técnica para la unidad se debe principalmente a la jornada laboral de treinta horas semanales. El NAS é um instrumento gerencial que posibilita adecuar el número de profesionales a las reales necesidades de cuidado de los pacientes, a fin de que sea realizado de modo seguro y con la cualidade ansiada.

PALABRAS CLAVE: Administración de personal; Enfermería; Cuidados críticos; Carga de trabajo.

\footnotetext{
'Enfermeira. Doutora em Engenharia de Produção. Professora Adjunta do Departamento de Enfermagem da Universidade Federal do Paraná - UFPR. Membro do Núcleo de Estudos, Pesquisa e Extensão em Cuidado Humano Enfermagem - NEPECHE/UFPR.

${ }^{2}$ Enfermeira. Especialista em Psicologia do Trabalho. Mestranda em Enfermagem pelo Programa de Pós-Graduação em Enfermagem da UFPR. Membro do NEPECHE/UFPR.

${ }^{3}$ Enfermeira. Hospital de Clinicas - HC/UFPR.

${ }^{4}$ Enfermeira do HC/UFPR. Especialista em Gestão Hospitalar. Mestre em Distúrbios da Comunicação. Docente. Universidade Tuiuti do Paraná.
}

Autor correspondente:

Lillian Daisy G. Wolff

Rua Padre Camargo, 120 - CEP 80060-240 - Curitiba-PR

E-mail: ldgw@ufpr.br

Recebido em: 02/07/07 Aprovado em: 07/08/07 


\section{INTRODUÇÃO}

O Hospital de Clínicas da Universidade Federal do Paraná (UFPR) é o maior hospital público do Paraná e importante centro de referência para a média e alta complexidade no Sistema Único de Saúde (SUS). Recentes mudanças em seu modelo organizacional concentraram as unidades de emergência e urgência em uma única unidade funcional, constituída por: Pronto Atendimento (PA), Centro de Terapia Semi-Intensiva (CTSI) e Unidade de Terapia Intensiva (UTI). O CTSI tem por finalidade atender pacientes clínicos semicríticos e críticos provenientes do PA. Dos seus vinte e cinco leitos, quinze destinam-se a pacientes semicríticos, seis a pacientes críticos da especialidade Neurologia e quatro para pacientes críticos da especialidade Cardiologia.

No decorrer das referidas mudanças, enfermeiras do CTSI observaram que seus pacientes passaram paulatinamente a apresentar necessidade de cuidados cada vez mais complexos, o que em sua percepção justifica o aumento da carga de trabalho referido pela equipe de enfermagem. Esta observação despertou-lhes a motivação para a realização de uma pesquisa a fim de testar a hipótese de que para o atendimento das necessidades de cuidado da clientela que interna no CTSI, segundo o padrão de qualidade que a unidade almeja manter, o número de profissionais de enfermagem lotado na unidade é inadequado.

Por conseguinte, estabeleceu-se uma parceria entre essas enfermeiras do CTSI e docentes do Departamento de Enfermagem da UFPR para o desenvolvimento desta pesquisa, cujo objetivo geral foi dimensionar profissionais de enfermagem de acordo com o perfil dos pacientes e a necessidade de cuidados nessa Unidade. Os objetivos específicos da pesquisa foram: i) descrever o perfil da clientela da Unidade; ii) analisar as atividades de enfermagem mais freqüentes no CTSI; iii) estimar a carga de trabalho de enfermagem segundo a complexidade dos cuidados de enfermagem requeridos pelos pacientes internados; e, iv) verificar se a idade e a especialidade pela qual o paciente interna têm relação com a complexidade e intensidade de cuidado que ele necessita receber.

Indubitavelmente, os hospitais têm procurado se modernizar, com vistas a utilizar racionalmente os recursos para prestar assistência de qualidade aos pacientes. Isso significa que além de recursos físicos, financeiros, tecnológicos e de informação, os hospitais necessitam, principalmente, de pessoas em número e qualificação apropriados para exercer atividades, com eficácia e eficiência.

Os hospitais visam atender as diferentes necessidades, manifestações e condições de saúde dos seus usuários e, em conseqüência disso, ofertam serviços tais como: cuidados, diagnóstico e tratamento, realizados por diferentes profissionais de saúde. À Enfermagem cabe a prestação de cuidados de manutenção e recuperação da vida, que variam de intensidade e complexidade de acordo com as necessidades, manifestações e condições do paciente. Para um adequado atendimento aos pacientes com crescentes níveis de complexidade de cuidados surgiram unidades voltadas à assistência intensiva, com evidente crescimento nas décadas de 60 e 70, denominadas como Unidades de Terapia Intensiva e Semi-Intensiva. A unidade de Terapia intensiva é:

\footnotetext{
Um serviço de internação para pacientes críticos que requerem atenção médica e de enfermagem permanente, com dotação própria de pessoal técnico e profissional especializado, com equipamentos específicos próprios e outras tecnologias destinadas ao diagnóstico e ao tratamento. São considerados pacientes críticos aqueles com desequilíbrio de um ou mais dos principais sistemas fisiológicos, com perda de sua auto-regulação mas potencialmente reversíveis ${ }^{(1: 1)}$.
}

Destacam-se entre as características peculiares de uma UTI: "o ambiente permeado por tecnologia de ponta, situações iminentes de emergência e necessidade constante de agilidade e habilidade no atendimento ao cliente"(2:331). No entanto, ainda são limitadas as informações disponíveis em nosso meio que indiquem quem são os pacientes que realmente necessitam de assistência intensiva ${ }^{(3)}$.

Por sua vez, a Unidade de Tratamento SemiIntensivo (Unidade Semi-Intensiva) se constitui de

[...] um conjunto de elementos funcionalmente agrupados, destinado ao atendimento de pacientes, preferencialmente oriundos da UTI, que requeiram cuidados de enfermagem intensivos e observação contínua, sob supervisão e acompanhamento médico, este último não necessariamente contínuo, porém linear ${ }^{(4: 2)}$.

A equipe de enfermagem dessas unidades necessita ter condições para conviver no seu processo de cuidar com "[...] situações emocionalmente conflitantes e de estresse (risco iminente de vida, a constante presença da morte, o convívio com a dor do outro)"(2:331), que acabam por influenciar sua própria 
situação de saúde. Some-se a essas situações a insuficiência de profissionais de enfermagem nessas unidades, $\mathrm{o}$ que impede $\mathrm{o}$ atendimento apropriado às necessidades dos pacientes e acarreta-lhes sobrecarga de trabalho.

Para que este cuidado de enfermagem tenha qualidade deve propiciar segurança aos pacientes, e por conseguinte, deve ser realizado por profissionais em número e qualificação condizente às necessidades da clientela da unidade.

A segurança dos pacientes no decorrer da assistência à saúde é um compromisso ético assumido pelos profissionais de enfermagem desde a sua formação e que encontra nos modelos gerenciais modernos instrumentos para a sua afirmação [...]. A ocorrência de eventos iatrogênicos na prática assistencial se contrapõe a esses princípios, visto que coloca em risco a vida do cliente/paciente ${ }^{(5: 18)}$.

Neste contexto, o dimensionamento de pessoal apresenta-se como uma ferramenta gerencial a ser utilizada pela enfermeira na

[...] etapa inicial do processo de provimento de pessoal, que tem por finalidade a previsão da quantidade de funcionários por categoria, requerida para suprir as necessidades de assistência de Enfermagem, direta ou indiretamente prestada à clientela ${ }^{(6: 126)}$.

Assim, a previsão adequada desses profissionais visa a prestação de cuidados com segurança e qualidade à clientela assistida.

Nos serviços de saúde é grande a rotatividade de profissionais de enfermagem, o que implica em grande número de rescisões de contrato e novas contratações, de outras despesas, horas de treinamento e de aperfeiçoamento( ${ }^{(7)}$. Portanto, a adequação dos integrantes dessa equipe, tanto no aspecto quantitativo como no qualitativo para o cuidado ao paciente, é necessária também para o gerenciamento adequado dos custos hospitalares.

Essa adequação de pessoal pode ser realizada por um método de dimensionamento de pessoal de enfermagem, que

consiste na aplicação de um processo sistemático para determinar o número e a categoria profissional requerida para prover os cuidados de enfermagem que garantam a qualidade, previamente estabelecida, a um grupo de pacientes $^{\left({ }^{(}\right)}$.

Desde 1998, Gaidzinski ${ }^{(8)}$ propôs um método de dimensionamento que requer o conhecimento da carga de trabalho da unidade, do Índice de Segurança Técnica (IST) e da jornada de trabalho da enfermagem.

Ademais, a Resolução COFEN 293/2004 ${ }^{(9)}$ fixa e estabelece parâmetros para dimensionamento do quadro de profissionais de enfermagem nos serviços de saúde e assemelhados, e tornou-se um instrumento que traz diretrizes às enfermeiras neste sentido. A resolução recomenda que o dimensionamento deve basear-se também em características relativas à instituição, aos serviços de enfermagem, às questões técnico-administrativas e ao tipo de clientela.

Segundo a referida resolução, compete à enfermeira estabelecer o quadro quantiqualitativo de profissionais necessário para a prestação do cuidado de enfermagem. Para a caracterização da clientela, a resolução preconiza a utilização de um Sistema de Classificação de Pacientes (SCP) que deve fornecer dados sobre a carga de trabalho de enfermagem requerida por paciente da unidade, nas 24 horas, segundo o tipo de assistência. A resolução também apresenta as horas mínimas de enfermagem requeridas por paciente, nas 24 horas, por tipo de cuidado, que foram identificadas a partir de estudos nacionais de dimensionamento de pessoal, e que constam no Quadro 1.

Quadro 1 - Horas de enfermagem por paciente nas 24 horas, segundo o tipo de assistência de enfermagem, de acordo com a Resolução 293/2004(9)

\begin{tabular}{|lc|}
\hline Tipo de Assistência & $\begin{array}{c}\text { Horas-enf / Paciente/ } \\
\mathbf{2 4} \text { horas }\end{array}$ \\
\hline Mínima ou Autocuidado & 3,8 \\
Intermediaria & 5,6 \\
Semi-intensiva & 9,4 \\
Intensiva & 17,9 \\
\hline
\end{tabular}

O Nursing Activities Score (NAS) é um Escore das Atividades de Enfermagem obtido a partir da avaliação dos cuidados requeridos aos pacientes críticos, como subsídio para o cálculo da carga de trabalho de Enfermagem. A metodologia do NAS foi traduzida e validada por Queijo ${ }^{(3)}$ para a língua portuguesa, em 2002. Segundo a autora ${ }^{(10)}$, o NAS abrange um conjunto maior de atividades desenvolvidas pela equipe de enfermagem do que os índices de gravidade de paciente anteriormente utilizados em Unidades de Terapia Intensiva para determinar a carga de trabalho de enfermagem, como o TISS-28 ${ }^{(11-12)}$. Queijo salienta que "entre outras 
utilidades, o NAS permite justificar ao administrador do hospital, por exemplo, a necessidade de pessoal adicional em terapia intensiva quando ocorre aumento da carga de trabalho, bem como subsidiar as decisões referentes ao recrutamento e seleção de pessoal de enfermagem" "(3:11). Portanto, por estas qualidades o NAS foi considerado apropriado para avaliar a carga de trabalho do CTSI.

\section{MÉTODO E TÉCNICAS}

A pesquisa descritiva-exploratória, de abordagem quantitativa foi realizada no Centro de Terapia Semi-intensiva (CTSI) do Hospital de Clínicas da Universidade Federal do Paraná (HC/UFPR). Seu projeto foi aprovado pelo Plenário do Departamento de Enfermagem, pelo Colegiado do Serviço de Emergências e Urgências Clínicas da UFPR, bem como pela Comissão de Ética do Hospital de Clínicas da UFPR, por estar em acordo com os preceitos da Resolução 196/96 ${ }^{(13)}$.

A amostra foi intencional e esperava-se contabilizar 100 pacientes, conforme o estudo de Queijo $^{(3)}$. Como quatro pacientes foram eliminados da amostra por falta de dados, a amostra final constituiu-se de noventa e seis pacientes. Os sujeitos foram selecionados a partir dos seguintes critérios: indivíduo de qualquer um dos sexos, com idade superior a quatorze (14) anos, internado na Unidade por um período mínimo de 24 horas, que consentiu a sua participação na pesquisa (ou seu responsável consentiu) durante todo o período de sua internação.

Os dados sobre a carga de trabalho e o grau de complexidade dos cuidados referentes a cada sujeito foram obtidos durante os meses de Outubro a Dezembro de 2004, mediante observação sistemática e preenchimento do instrumento em anexo, cuja primeira parte refere-se à identificação do paciente (iniciais do nome, idade, procedência, e demais dados sobre sua internação), e a segunda parte com os itens do NAS (em anexo). A atividade de coleta de dados ficou a cargo das enfermeiras pesquisadoras do CTSI e de alunas voluntárias do Curso de Graduação em Enfermagem da UFPR, com treinamento prévio e acompanhamento. Os dados de cada paciente foram obtidos uma vez ao dia, no mesmo horário, durante todo o período de sua internação. Por sua vez, os dados relativos às ausências previstas e não previstas, necessários para o cálculo do Índice de Segurança Técnica, foram coletados mediante o levantamento dessas ausências nos registros das escalas de trabalho da Unidade, no ano de 2004.

Todos os dados foram organizados em planilhas eletrônicas. Os dados de identificação foram submetidos à análise descritiva univariada, e calculada a sua freqüência relativa. Também foram calculadas a freqüência absoluta e relativa de cada intervenção listada no instrumento, de modo a caracterizar os cuidados mais freqüentes realizados com os pacientes da amostra, no período de coleta de dados. Procedeu-se à análise descritiva univariada dos escores NAS, assim como à análise bi-variada entre estes e a variável idade dos pacientes.

\section{RESULTADOS}

\section{O perfil da clientela do CTSI}

Entre os 96 pacientes, 54\% eram do sexo masculino e $48 \%$ do sexo feminino. A maioria era residente em Curitiba (59\%) e região metropolitana (11\%), mas houve $3 \%$ que residiam no interior do estado e $1 \%$ em outro estado. Esses dados aproximam-se dos dados de procedência de pacientes internados em todo o Hospital de Clínicas, conforme pode ser observado no Quadro 2, referente ao período de 2003 a 2005 . Essa distribuição de pacientes, segundo o local de residência, caracteriza o fato de que o HC/UFPR é um hospital de referência de alta complexidade para o SUS em Curitiba e Região metropolitana.

Quadro 2 - Procedência dos pacientes atendidos, Hospital de Clínicas da UFPR, 2003-2005

\begin{tabular}{|llll|}
\hline Região de proce dência & $\mathbf{2 0 0 3}$ & $\mathbf{2 0 0 4}$ & $\mathbf{2 0 0 5}$ \\
\hline Curitiba & $53 \%$ & $54 \%$ & $56 \%$ \\
Região Metropolitana & $26 \%$ & $24 \%$ & $29 \%$ \\
Outros Municípios - Paraná & $17 \%$ & $17 \%$ & $11 \%$ \\
Total Paraná & $96 \%$ & $95 \%$ & $96 \%$ \\
Outros Estados & $4 \%$ & $5 \%$ & $4 \%$ \\
Outros Países & $0,003 \%$ & $0,01 \%$ & $0,01 \%$ \\
\hline
\end{tabular}

Fonte: Hospital de Clínicas/UFPR ${ }^{(14)}$

Os pacientes do CTSI que participaram da pesquisa procederam, prioritariamente, do Pronto Atendimento (76\%), uma vez que é a principal porta de entrada do HC/UFPR para o atendimento de 
urgências e emergências. Contudo, houve pacientes que ingressaram no CTSI por meio de transferência da Unidade de Clínica Médica (9\%), da UTI (8\%), da Infectologia (2\%), do Serviço de Hemodinâmica (2\%), da Unidade de Clínica Cirúrgica (1\%), do Centro Cirúrgico (1\%), e ainda de outro hospital (1\%). Essas transferências indicam que a Unidade recebeu pacientes que estiveram internados em diversas unidades e que foram encaminhados para o CTSI quando suas condições clínicas se agravaram, e passaram a necesitar de cuidados intensivos ou semiintensivos. Ocorreram também transferências da UTI, provavelmente quando as condições clínicas desses pacientes melhoraram, mas eles não tinham condições de serem transferidos para outras unidades de internação. Esta situação retrata a alta taxa de ocupação da UTI deste hospital, superior a 100\% no ano de 2004 e subseqüentes ${ }^{(14)}$.

Quanto ao tipo de saída do paciente internado no CTSI, evidenciou-se uma ocorrência de $28 \%$ de óbitos, $11 \%$ transferidos para a UTI, 3\% para o Centro Cirúrgico e 1\% para o PA observação. Essa situação denota a gravidade do estado clínico desses pacientes. Contudo, 29\% dos pacientes receberam alta e outros $28 \%$ foram transferidos para outras unidades de internação, situação que retrata o papel do CTSI como uma unidade que restaura as condições dos pacientes críticos e semicríticos e os encaminha para unidades de assistência de menor complexidade.

Durante o período de coleta de dados, constatou-se que o processo de trabalho da enfermagem no CTSI era realizado por 07 enfermeiras, 61 auxiliares de enfermagem e 03 técnicos em enfermagem, dispostos em três turnos de trabalho: manhã, tarde e noite. O método de organização do trabalho de enfermagem adotado na unidade era o método integral, no qual cada profissional é responsável pelo provimento de todos os cuidados que o paciente necessita. Esse método foi adotado em virtude da necessidade de aprimorar a qualidade da assistência ao paciente, pois possibilita o seu acompanhamento pela Enfermagem de modo ininterrupto em um turno de trabalho. Contudo, não há distinção das atribuições do técnico e do auxiliar de enfermagem. Ao enfermeiro chefe da unidade cabe funções administrativas, e aos demais enfermeiros, o gerenciamento da assistência e atividades de cuidado de maior complexidade.

A aplicação do NAS permitiu a identificação dos cuidados requeridos aos pacientes a partir de suas necessidades individuais e suas condições clínicas, considerando cada intervenção prevista no instrumento. A análise dos dados de todos os pacientes da amostra possibilitou a classificação das atividades que requerem maior carga de trabalho dos profissionais de enfermagem no CTSI. Em primeiro lugar, ficou o Item $6 \mathrm{~b}$, relativo à "Realização de procedimentos de mobilização e posicionamento mais do que 3 vezes em 24 horas ou com dois enfermeiros em qualquer freqüência", e que exigiu, em média, 2 horas por paciente/dia. Em segundo lugar ficou o Item 3, relativo à "Medicação, exceto drogas vasoativas," e que exigiu, em média, 1 hora e 17 minutos por paciente/dia; em terceiro ficou o Item 17, relativo à "Medida quantitativa do débito urinário (sonda vesical de demora)", e que exigiu, em média, 1 hora e 11 minutos por paciente/ dia.

Por sua vez, os itens que se apresentaram em $4^{\circ}$ lugar como os que mais demandavam horas de assistência em enfermagem foram: o Item 2 , relativo à "Investigações laboratoriais", que exigiam 1,0 hora por paciente/dia, e o Item 8, relativo à "Realização de Tarefas Administrativas e Gerenciais de Rotina, como: processamento de dados clínicos, solicitação de exames, troca de informações profissionais (passagem de plantão, visitas clínicas)", que também exigiam 1,0 hora por paciente/dia.

No grupo de "Monitorização e controles", a média de horas demandadas por paciente/dia foi de 1 hora, e no grupo de "Procedimentos de Higiene", a média de horas demandadas por paciente/dia foi de 1 hora e 38 minutos. Os itens do NAS que demandaram mais horas de enfermagem totalizaram 9 horas e 6 minutos de assistência ao paciente.

A partir dos cálculos dos valores do NAS referentes aos 96 pacientes internados no CTSI, foi possível estimar a carga de trabalho de enfermagem e realizar o cálculo de pessoal para essa Unidade, mediante a fórmula proposta por Gaidzinski ${ }^{(15)}$, cujos compomponentes do Índice de Segurança Técnica ${ }^{(6)}$ estão apresentados no Quadro 3.

A média do NAS, relativa a todos os pacientes do CTSI participantes do estudo, foi de 45,85 e o desvio padrão foi igual a 15 . O valor da média aproximou-se do valor da mediana $(45,1)$, e da moda $(43,5)$, sendo que este foi o valor mais repetido entre os NAS. Essa situação confere à distribuição dos valores em torno da média com características de uma curva normal, conforme pode ser observado na Figura 1, e indica que a média é representativa da amostra. 
$\mathrm{Q}=\frac{\left(\mathrm{NAS}+\mathrm{S}_{\mathrm{NAS}}\right)}{100} * \frac{24}{\mathrm{t}}\left\{\left(\left[1+\frac{\mathrm{E} \%}{100}\right) *\left(1+\frac{\mathrm{F} \%}{100}\right) *\left(1+\frac{\mathrm{V} \%}{100}\right) *\left(1+\frac{\mathrm{A} \%}{100}\right)\right]\right\}$

em que:

$Q=$ quantidade total de pessoal de enfermagem no ano de 2004;

$N A S=$ média do escore NAS calculado para o CTSI;

$S_{\mathrm{NAS}}=$ desvio padrão do escore NAS calculado;

$E \%=$ percentual de ausências por folga semanal no ano de 2004;

$F \%=$ percentual de ausências por dias feriados no ano de 2004;

$V \%=$ percentual de ausências por férias dos profissionais de enfermagem no ano de 2004;

$A \%=$ percentual de ausências não previstas dos profissionais de enfermagem no ano de 2004 .

$t=$ tempo efetivo de trabalho (cuidado direto e indireto prestado ao paciente, por turno).

Quadro 3 - Componentes do Índice de Segurança Técnica

\begin{tabular}{|c|c|c|c|c|c|}
\hline $\begin{array}{l}\text { Componentes do índice de } \\
\text { egurança técnica (IST) }\end{array}$ & $\begin{array}{ll}\text { Ausências previstas por } & \text { Ausências previstas } \\
\text { folga semanal }(\mathrm{E} \%) & \text { por feriado }(\mathrm{F} \%)\end{array}$ & $\begin{array}{l}\text { Ausências previstas } \\
\text { por férias }\left(\mathrm{V}_{\mathrm{K}} \%\right)\end{array}$ & $\begin{array}{l}\text { Ausên } \\
\text { previs }\end{array}$ & $\operatorname{tas}\left(A_{k}\right.$ & \\
\hline Fórmulas & $E \%=\frac{f}{D-f} \cdot 100$ & $V_{k}=\frac{v_{k}}{D-v_{k}} \cdot 100$ & $A \%=$ & $\mid \frac{\sum_{l} a_{k i}}{D-\Sigma a_{k i}}$ & $\cdot 100$ \\
\hline \multicolumn{6}{|c|}{$\begin{array}{l}\text { em que: } \\
e=\text { número de dias de folga, por semana, dos trabalhadores de enfermagem; } \\
d=\text { número de dias trabalhados na unidade; } \\
f=\text { dias feriados no ano; } \\
\mathrm{D}=\text { dias do ano ( } 365 \text { dias); } \\
v_{k}=\text { média de dias de férias da categoria profissional }{ }_{\mathrm{k}} \text { (enfermeiro, técnico e auxiliar); } \\
\sum a_{k, i} \text { somatório dos dias médios de ausências não previstas, segundo os tipos de ausências (faltas, licenças e } \\
\text { suspensões) por categoria profissional }(\text { (enfermeiro, técnico e auxiliar). }\end{array}$} \\
\hline
\end{tabular}

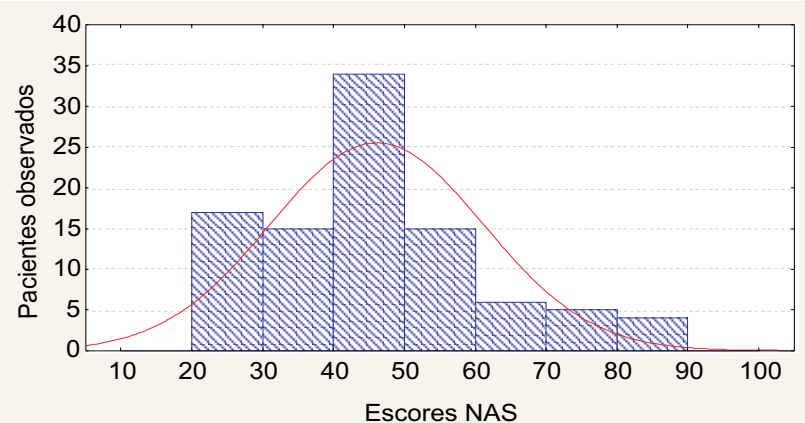

Figura 1 - Histograma dos valores do NAS para o CTSI do Hospital de Clínicas da UFPR, 2004, amostra de 96 pacientes

$\mathrm{Na}$ análise dos valores do NAS, obteve-se uma carga de trabalho de enfermagem para o CTSI de 14,60 horas de assistência por paciente, nas 24 horas. Esta carga de trabalho implica que cada paciente requer 4 profissionais de enfermagem para cuidá-1o nas 24 horas, considerando turnos de trabalho de 6 horas.
A análise bivariada entre a variável idade e o escore NAS identificou uma correlação de 0,09 , que indica que a média do valor do NAS obtido dos pacientes do CTSI, nesse estudo, não sofreu influência da idade dos pacientes. Isto pode ser comprovado pelos dados apresentados na Tabela 1 , em que se verifica que $25 \%$ dos pacientes observados tinham entre 16 e 40 anos, e nesta faixa de idade o valor médio do NAS foi de 15,52.

A maioria dos pacientes $(50 \%)$ tinha idade entre 41 a 69 anos, e nesta faixa o valor médio do NAS foi de 15,05 horas/paciente/24 horas. Os restantes $25 \%$ dos pacientes tinham idade entre 70 a 94 anos, e o valor médio do NAS nesta faixa etária foi de 14,01. Não foram constatadas variações relevantes entre o valor médio do NAS para essas três faixas etárias, portanto, independemente da idade, cada um requeria quatro profissionais de enfermagem para cuidá-lo, nas 24 horas.

A idade não importa quando o paciente apresenta múltiplos eventos, pois nestes casos é grande o número de problemas, o que aumenta a 
chance de ocorrer complicações e morte ${ }^{(12)}$. A relação entre os quartis por idade e a carga de trabalho de enfermagem pode ser vista na Tabela 1.

Tabela 1 - Demonstrativo da distribuição quartil da variável idade, e respectivas cargas de trabalho da enfermagem (horas de enfermagem por paciente nas 24 horas) no CTSI do Hospital de Clínicas da UFPR, 2004

\begin{tabular}{lr}
\hline $\begin{array}{l}\text { Distribuição quartil } \\
\text { por Idade }\end{array}$ & $\begin{array}{r}\text { Horas-enf/ Paciente } \\
\text { /24 horas }\end{array}$ \\
\hline$(25 \%) 16$ a 40 anos & 13,52 \\
$(50 \%) 41$ a 69 anos & 15,09 \\
$(25 \%) 70$ a 94 anos & 14,01 \\
\hline
\end{tabular}

Outrossim, considerando as Horas-enf/ Paciente/dia mínimas, preconizadas pela Resolução $293 / 2004^{(9)}$ para cada tipo de assistência de enfermagem, apresentadas no Quadro 1, pode-se classificar o grau de complexidade dos pacientes do CTSI como variando entre semi-intensivo e intensivo.

A Tabela 2 indica que houve variação nos valores médios do NAS, ao serem considerados o tipo de leito (intensivo e semi-intensivo) e a especialidade médica (Neurologia, Cardiologia, Clínica Médica) pela qual o paciente foi admitido no CTSI. Constatouse que os graus de complexidade dos cuidados dos pacientes obtidos pelo NAS apresentaram relação com a especialidade pela qual foi internado o paciente. Se for considerada a média dos valores do NAS de todos os pacientes internados no CTSI, e a média dos pacientes internados em leitos para pacientes críticos da Cardiologia, observa-se que estes necessitavam de 4 profissionais de enfermagem para prestarem os cuidados requeridos nas 24 horas. Por sua vez, se for considerada a média dos valores do NAS dos pacientes internados em leitos de cuidado intensivo da Neurologia, e a dos internados em leitos de cuidado semi-intensivo da Clínica Médica, observa-se que estes necessitavam de 5 profissionais/paciente, nas 24 horas.

O Índice de Segurança Técnica calculado para o CTSI no valor de 0,65 , é considerado um valor altíssimo. Ele é constituído pela multiplicação dos índices de folgas $(1,40)$, feriados $(1,061)$, férias $(1,06)$ e ausências não previstas por atestado e licença médicas (1,0564). A Resolução 293/2004(9) recomenda que ele seja de, no mínimo, 15\%. No caso do CTSI, esse alto índice deve-se principalmente pelo fato da jornada de trabalho semanal ser de 30 horas e o hospital conceder duas folgas semanais a cada profissional.

Tabela 2 - Tipo de leito, especialidade médica, valor médio do NAS, carga de trabalho da enfermagem (horas de enfermagem por paciente nas 24 horas) e número de profissionais calculados por paciente, nas 24 horas, CTSI do Hospital de Clínicas da UFPR, 2004, amostra de 96 pacientes

\begin{tabular}{lccc}
\hline Tipo de leito $x$ & Valor & $\begin{array}{c}\text { Horas de } \\
\text { enfermagem } \\
\text { especialidade }\end{array}$ & $\begin{array}{c}\mathbf{N}^{\circ} \text { de } \\
\text { profissionais } \\
\text { enfermagem }\end{array}$ \\
médica & NAS & $/ 24$ horas & $\begin{array}{c}\text { /paciente/24 } \\
\text { horas }\end{array}$ \\
\hline
\end{tabular}

\section{Leitos de}

cuidado semi-

intensivo (Clínica

Médica) $\quad 65,88 \quad 15,81 \quad 4,45=>5$

Leitos de

cuidado intensivo

(Neurologia)

67,5

16,20

$4,5=>5$

Leitos de

cuidado intensivo

(Cardiologia)

55,62

13,35

$3,7=>4$

Leitos de

cuidado intensivo

(de ambas as

especialidades)

$$
59,63
$$

14,30

$3,95=>4$

Leitos de

cuidado semi-

intensivo de

todos os tipos e

especialidade

60,85

14,60

4,0

Após a obtenção das variáveis necessárias para a estimativa do Índice de Segurança Técnica, foram realizados os cálculos para o dimensionamento de pessoal. O Quadro 4 demonstra o quadro de pessoal existente na unidade, e o quadro de pessoal projetado segundo a proporção das categorias profissionais existentes. Observa-se que seriam necessários mais 29 funcionários (41\% a mais) do que já existia na Unidade, para que fossem supridas as necessidades de cuidado dos pacientes internados no CTSI, considerando a amostra dos pacientes avaliados neste estudo.

Quadro 4 - Demonstrativo do quadro de pessoal de enfermagem existente, e projetado segundo a proporção atual das categorias, Hospital de Clínicas da UFPR, 2004 


\begin{tabular}{|lcc|}
\hline $\begin{array}{l}\text { Categoria } \\
\text { Profissional }\end{array}$ & $\begin{array}{c}\text { Quadro de } \\
\text { pessoal } \\
\text { existente }\end{array}$ & $\begin{array}{c}\text { Quadro de pessoal } \\
\text { projetado segundo a } \\
\text { proporção das categorias }\end{array}$ \\
\hline Enfermeiro & $7(10 \%)$ & $10(10 \%)$ \\
Técnico & $3(4 \%)$ & $4(4 \%)$ \\
Auxiliar & $61(86 \%)$ & $86(86 \%)$ \\
Total & $71(100 \%)$ & $100(100 \%)$ \\
\hline
\end{tabular}

De acordo com a Resolução 293/2004(9), os pacientes de cuidado semi-intensivo necessitam de, no mínimo, 9,4 horas de enfermagem/24 horas, enquanto que os pacientes de cuidados intensivos necessitam de, no mínimo, 17,9 horas de enfermagem/ 24 horas. De acordo com o NAS, os pacientes do CTSI necessitam em média de, no mínimo, 14,60 horas/ paciente/24 horas. Constatou-se que os pacientes internados no CTSI efetivamente dependem da Enfermagem para cuidados de manutenção e restauração da vida, cuidados contínuos e complexos e que demandam uma carga de trabalho que se situa entre os cuidados intensivos e semi-intensivos. Para dar conta dessa carga de trabalho seria necessário um quadro de pessoal com mais 29 funcionários (41\%) do que existia na Unidade na época em que foram coletados os dados para esta pesquisa. Pode-se inferir que os funcionários do CTSI não estavam trabalhando em número adequado para prestar aos pacientes internados os cuidados que estes requeriam, de acordo com as suas necessidades e condições clínicas. Essa situação pode ter contribuído para o estresse, desmotivação dos funcionários e exposição dos pacientes a riscos relacionados à sua segurança e integridade.

\section{CONSIDERAÇÕES FINAIS}

A complexidade e a intensidade do cuidado aos pacientes, identificadas pelos valores obtidos do NAS, não sofrem influência da idade do paciente, mas têm relação com a especialidade pela qual este foi internado, são influenciadas pelas suas condições clínicas e pela dependência das atividades de profissionais de enfermagem. Os resultados evidenciaram que a clientela internada nesta Unidade, no momento da realização da pesquisa, requeria cuidados contínuos e complexos que se traduziram em carga de trabalho excessiva para o número de profissionais que ali trabalhavam. Assim, foi corroborada a hipótese de que para o atendimento das necessidades de cuidado da clientela que interna no CTSI, constituída de pacientes de cuidados semiintensivos e intensivos, o número de profissionais de enfermagem lotado na unidade é inadequado para o padrão de qualidade que as enfermeiras da Unidade almejam.

Os pacientes internados nos leitos destinados a pacientes semi-intensivos da Clínica Médica têm, em média, maior demanda por horas de enfermagem do que os pacientes da Cardiologia, internados em leitos para cuidados intensivos para esta especialidade. Isso se explica, pois o motivo de internação mais freqüente nesta especialidade no CTSI é o Infarto Agudo do Miocárdio. Neste caso, o paciente fica continuamente sob cuidados da enfermagem, mas muitas vezes encontra-se consciente e capaz de realizar algumas ações que o tornam menos dependente dos profissionais.

Contudo, os pacientes internados em leitos para cuidados intensivos destinados à Neurologia apresentaram maior demanda de cuidados de enfermagem, devido ao fato de o principal motivo de internação nessa especialidade ser por seqüelas de Acidente Vascular Cerebral. O paciente acometido por esta condição, geralmente está com muitas restrições motoras e psíquicas, e por conseguinte, é mais dependente das ações de enfermagem.

Ademais, em 2004 a média mensal de internação de pacientes em leitos intensivos da Cardiologia no CTSI foi 35, e o tempo de permanência médio de 5,02 dias, em contraposição a uma média de 7 pacientes internados, e uma média de permanência de 8,05 dos pacientes internados pela Neurologia.

A associação da condição do paciente com a dependência da Enfermagem e a carga de trabalho demandada no seu cuidado deve ser levada em conta no planejamento do cuidado na Unidade, com vistas à reorganização do processo de trabalho no CTSI, a fim de que: (i) seja evitada a sobrecarga de trabalho, mediante o uso eficiente dos recursos humanos na distribuição diária de atividades de seus profissionais; e além disso, que (ii) ocorra melhoria das condições de trabalho e (iii) a colaboração dos demais profissionais da equipe de saúde da Unidade. Adicionalmente, a análise de atividades de cuidado de enfermagem mais freqüentes no CTSI também pode auxiliar o planejamento da proporcionalidade de profissionais de enfermagem necessários segundo a categoria profissional.

É recomendável que esta pesquisa seja desenvolvida sistematicamente, no período de trinta dias, em meses típicos da produção da Unidade. Neste 
caso, é necessário que seja escolhido um mês do ano no qual geralmente o número de internações no CTSI se aproxime da média anual. Sendo assim, devem ser evitados, por exemplo, os meses de férias da equipe médica, em que ocorrem menos internações. Novas pesquisas possibilitarão verificar se o perfil da clientela e suas necessidades de cuidado de enfermagem modificam-se a ponto de influir na carga de trabalho de enfermagem calculada nesta pesquisa, e fornecerão subsídios importantes para que a administração do hospital tome medidas que viabilizem a eficiência e eficácia da assistência.

Outros estudos de dimensionamento de recursos humanos de enfermagem podem ser realizados, mas que contemplem também a análise do ambiente interno e externo à unidade em estudo, a fim de que sejam identificadas outras variáveis que possam intervir no trabalho da Enfermagem.

Recomenda-se, também, que novos estudos sejam realizados por meio da parceria entre enfermeiras do serviço e docentes de Enfermagem, uma vez que se constataram benefícios para ambas as partes no desenvolvimento desta pesquisa. Reconhecese que as enfermeiras do serviço são as que têm melhor condição de avaliar as necessidades de cuidado de seus clientes, de acordo com o seu planejamento e com a qualidade almejada. Em contrapartida, as pesquisadoras docentes detêm conhecimentos e habilidades em desenvolver projetos de pesquisa, que ao serem compartilhados com o serviço aproximam a teoria à prática, e promovem o desenvolvimento científico da Enfermagem.

\section{REFERÊNCIAS}

1 Ministério da Saúde (BR). Gabinete do Ministro. Portaria $\mathrm{n}^{\mathrm{o}} 551$, de 13 de abril de 2005. Aprova o documento Requisitos Comuns para Unidades de Terapia Intensiva de Adultos do MERCOSUL. Diário Oficial da União. Brasília,2005. n.71.

2 Bolela F, Jerico MC. Unidades de terapia intensiva: considerações da literatura acerca das dificuldades e estratégias para sua humanização. Esc Anna Nery. 2006;10(2):301-309.

3 Queijo AF. Tradução para o português e validação de um instrumento de medida de carga de trabalho de enfermagem em Unidade de Terapia Intensiva: Nursing Activities Score(NAS) [dissertação] . São Paulo (SP): Escola de Enfermagem, Universidade de São Paulo; 2002.

4 Ministério da Saúde (BR). Secretaria de Vigilância Sanitária. Portaria n ${ }^{\circ}$ 466/MS/SVS de 04 de Junho de
1998. Diário Oficial da União. Brasília, 1998. Disponível em: http://www.anvisa.gov.br/legis/portarias/ 466_98.htm. (12 Jun 2007)

5 Padilha KG. Ocorrências iatrogênicas em Unidade de Terapia Intensiva (UTI): análise dos fatores relacionados. Rev Paul Enferm. 2006 Mar;25(1):18-23.

6 Gaidzinski RR., Fugulin FMT; Castilho, V. Dimensionamento de pessoal de enfermagem em instituições de saúde. In: Kurcgant P., organizador. Gerenciamento em enfermagem. Rio de Janeiro: Guanabara Koogan; 2005:125-37.

7 Antunes AV, Costa MN. Dimensionamento de pessoal de enfermagem em um Hospital Universitário. Rev Latino-Am Enferm. 2003;11(6):832-9

8 Gaidzinski RR. Dimensionamento de pessoal de enfermagem em instituições hospitalares [tese]. São Paulo (SP): Escola de Enfermagem, Universidade de São Paulo; 1998.

9 Conselho Federal de Enfermagem. Resolução n. 293 de 21 de setembro de 2004. Estabelece os parâmetros para dimensionar o quantitativo dos diferentes níveis de formação dos profissionais de Enfermagem para a cobertura assistencial nas instituições de saúde. Disponível em: http://www.portalcofen.gov.br. (15 Mai 2007).

10 Queijo A. Nursing Activities Score (NAS) - Uma ferramenta para mensurar carga de trabalho da enfermagem em UTI. In: Dimensionamento de pessoal de enfermagem em hospitais: aspectos qualiquantitativos, 2006, Curitiba. Disponível em: http:// www.enfermagem.ufpr.br/evento_dimens.htm.(14 Abr. 2006)

11 Ministério da Saúde (BR). Resolução no ${ }^{\circ} 196$ de 10 de Outubro de 1996, sobre Diretrizes e Normas Regulamentadoras de Pesquisa envolvendo Seres Humanos do Ministério de Saúde. Disponível em: http:/ /www.conselho.saude.gov.br/resolucoes/1996/ Reso196.doc. (10 Fev 2006)

12 Elias ACGP, Matsuo T, Cardoso LTQ, Grion, CMC. Aplicação do sistema de pontuação de intervenções terapêuticas (TISS 28) em Unidade de Terapia Intensiva para a avaliação da gravidade do paciente. Rev Latinoam Enferm. 2006;14(3):324-29.

13 Miranda DR, Nap R, De Rijk A, Schaufeli W, Iapichino G. Nursing Activities Score. Crit Care Med. 2003;31(2):374-382.

14 Universidade Federal do Paraná. Hospital de Clínicas.Folder 2006. Disponível em http:// www.hc.ufpr.br/folder $2006 /$ atuaca o/ assistencia.html\#atendambu. (12 Jun 2007)

15 Gaidzinski RR. Dimensionamento de pessoal: evolução e tendências. In: Dimensionamento de pessoal de Enfermagem em hospitais: aspectos qualiquantitativos, 2006, Curitiba. Disponível em: http:// www.enfermagem.ufpr.br/evento_dimens.htm.(14 Abr 2006) 
Instrumento de coleta de dados

HOSPITAL DE CLÍNICAS - UFPR - CENTRO DE TERAPIA SEMI-INTENSIVA

\section{I - Identificação}

Iniciais:

Idade:

Sexo: ( ) M ( ) F

Residência: ( ) Curitiba - Pr ( ) Reg. Metropolitana ( ) Outro Estado ( ) Outro País

\section{II - Dados de internação}

Data:

Procedência:
( ) $\mathrm{CM}$
( ) Centro cirúrgico
( ) P.A
( ) Unid. Semi-intensiva
( ) Clínica cirúrgica
( ) Outro

III - Tipo de internação:

( ) Clínica médica ( ) Cardiologia ( ) Neurologia ( ) Cirúrgica

IV - Diagnóstico - CID:

\section{V - Doença crônica:}

( ) Hepática ( ) Cardiovascular ( ) Respiratória ( ) Renal ( ) Imunocomprometido

VI - Transferência da U.S.I - Data: __ _

( ) Unid. Internação ( ) Óbito ( ) Transferência ( ) UTI ( ) Outro

VII - Alta - Data:

NURSING ACTIVITIES SCORE (NAS)

\begin{tabular}{|c|c|c|c|}
\hline Atividades básicas & Pontuação & $1^{\circ}$ dia & $2^{\circ}$ dia \\
\hline $\begin{array}{l}\text { 1. Monitorização e controles } \\
\text { 1.a Sinais vitais horários, cálculo e registro regular do balanço hídrico (registro de ingesta/excreta). }\end{array}$ & 4,5 & & \\
\hline $\begin{array}{l}\text { 1.b Presença à beira do leito e observação ou atividade contínua por } 2 \text { horas ou mais, em } \\
\text { algum plantão por razões de segurança, gravidade ou terapia, tais como: ventilação mecânica } \\
\text { não-invasiva, desmame, agitação, confusão mental, posição prona, procedimentos de doação de } \\
\text { órgãos, preparo e administração de fluidos ou medicação, auxilio em procedimentos específicos. }\end{array}$ & 12,1 & & \\
\hline $\begin{array}{l}\text { 1.c Presença à beira do leito e observação ou atividade contínua por } 4 \text { horas ou mais, em } \\
\text { algum plantão por razões de segurança, gravidade ou terapia, tais como os exemplos acima. }\end{array}$ & 19,6 & & \\
\hline
\end{tabular}

Continua

Cogitare Enferm. 2007 Abr/Jun; 12(2):171-82 


\begin{tabular}{|c|c|c|c|}
\hline 2. Investigações laboratoriais: bioquímicas e microbiológicas. & 4,3 & & \\
\hline 3. Medicação, exceto drogas vasoativas. & 5,6 & & \\
\hline $\begin{array}{l}\text { 4. Procedimentos de higiene: } \\
\text { 4.a Realização de procedimentos de higiene tais como: curativo de feridas e } \\
\text { cateteres intravasculares, troca de roupa de cama, higiene corporal do paciente em } \\
\text { situações especiais (incontinência, vômito, queimaduras, feridas com secreção, } \\
\text { curativos cirúrgicos complexos com irrigação), procedimentos especiais (ex. } \\
\text { isolamento), etc. }\end{array}$ & 4,1 & & \\
\hline $\begin{array}{l}\text { 4.b Realização de procedimentos de higiene que durem mais do que } 2 \text { horas, em } \\
\text { algum plantão. }\end{array}$ & 16,5 & & \\
\hline $\begin{array}{l}\text { 4.b Realização de procedimentos de higiene que durem mais do que } 4 \text { horas, em } \\
\text { algum plantão. }\end{array}$ & 20 & & \\
\hline \multirow[t]{2}{*}{ 5. Cuidados com drenos todos (exceto sonda gástrica). } & 1,8 & & \\
\hline & Pontuação & $1^{\circ}$ dia & $2^{\circ}$ dia \\
\hline $\begin{array}{l}\text { 6. Mobilização e posicionamento incluindo procedimentos tais como: mudança de } \\
\text { decúbito, mobilização do paciente; transferência da cama para a cadeira; mobilização } \\
\text { do paciente em equipe (exemplo de paciente imóvel, tração, posição prona) } \\
\text { 6.a Realização do(s) procedimento(s) até } 3 \text { vezes, em } 24 \text { horas. }\end{array}$ & 5,5 & & \\
\hline $\begin{array}{l}\text { 6.b Realização do(s) procedimento(s) mais do que } 3 \text { vezes, em } 24 \text { horas, ou com } \\
\text { dois enfermeiros em qualquer frequiência. }\end{array}$ & 12,4 & & \\
\hline $\begin{array}{l}\text { 6.c Realização do(s) procedimento(s) com } 3 \text { ou mais enfermeiros em qualquer } \\
\text { frequiência. }\end{array}$ & 17,0 & & \\
\hline $\begin{array}{l}\text { 7. Suporte e cuidados aos familiares e pacientes incluindo procedimentos tais como: } \\
\text { telefonemas, entrevistas, aconselhamento. Freqüentemente, o suporte e cuidado, } \\
\text { sejam aos familiares ou aos pacientes, permitem à equipe continuar com outras } \\
\text { atividades de enfermagem (ex.: comunicação com os familiares enquanto presente à } \\
\text { beira do leito observando o paciente). } \\
\text { 7.a Suporte e cuidado aos familiares e pacientes que requerem dedicação exclusiva } \\
\text { por cerca e uma hora, em algum plantão, tais como: explicar condiçôs clínicas, lidar } \\
\text { com a dor e angústia, lidar com circunstâncias familiares difíceis. }\end{array}$ & 4,0 & & \\
\hline $\begin{array}{l}\text { 7.b Suporte e cuidado aos familiares e pacientes que requerem dedicação exclusiva } \\
\text { por } 3 \text { horas ou mais, em algum plantão, tais como: circunstâncias trabalhosas (ex: } \\
\text { grande número de familiares, problemas de linguagem, familiares hostis). }\end{array}$ & 32,0 & & \\
\hline \multicolumn{4}{|l|}{ 8. TAREFAS ADMINISTRATIVAS E GERENCIAIS } \\
\hline $\begin{array}{l}\text { 8.a. Realização de tarefas de rotinas tais como: processamento de dados clínicos, } \\
\text { solicitação de exames, troca de informações profissionais (por ex: passagem de } \\
\text { plantão, visitas clínicas). }\end{array}$ & 4,2 & & \\
\hline $\begin{array}{l}\text { 8.b. Realização de tarefas administrativas e gerenciais que requerem dedicação } \\
\text { integral, por cerca de } 2 \text { horas, em algum plantão, tais como: atividades de pesquisa, } \\
\text { aplicação de protocolos, procedimentos de admissão e alta. }\end{array}$ & 23,2 & & \\
\hline SUPORTE VENTILATÓRIO & & & \\
\hline
\end{tabular}




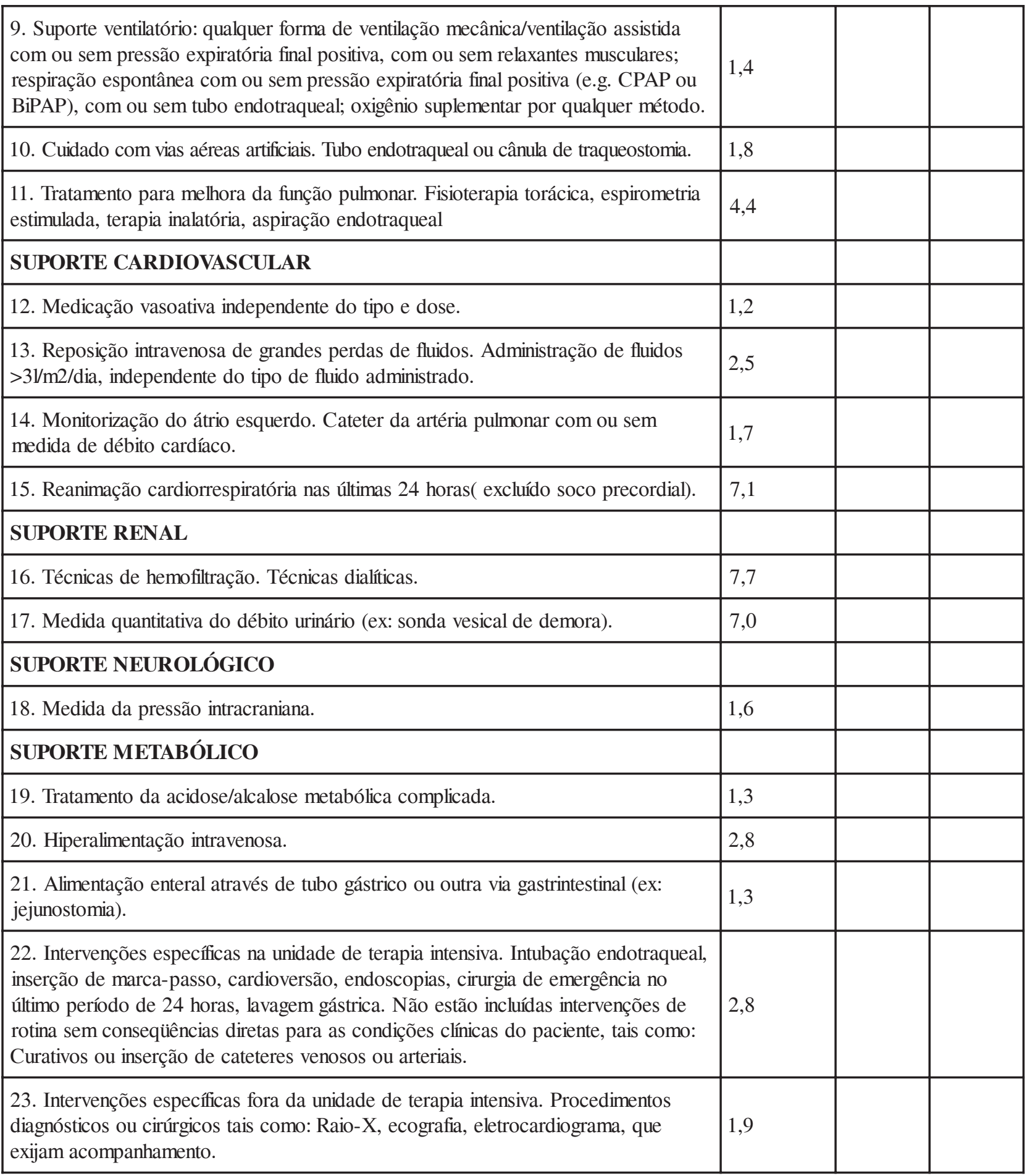

Os sub-itens dos itens 1,4,6,7 e 8 são mutuamente exclusivos. 